\title{
Bulk electronic structure of silicon studied with angle-resolved photoemission from the $\mathrm{Si}(100) 2 \times 1$ surface
}

\author{
L. S. O. Johansson* and P. E. S. Persson ${ }^{\dagger}$ \\ Department of Physics and Measurement Technology, Linköping Institute of Technology, S-581 83 Linköping, Sweden \\ U. O. Karlsson \\ Max-lab Synchrotron Radiation Group, P.O. Box 118, University of Lund, S-221 00 Lund, Sweden \\ R. I. G. Uhrberg \\ Department of Physics and Measurement Technology, Linköping Institute of Technology, S-581 83 Linköping, Sweden
}

(Received 24 April 1990)

\begin{abstract}
The bulk band structure of silicon along the $\Delta$ symmetry line in the Brillouin zone has been studied with polarization-dependent angle-resolved photoemission from the $\mathrm{Si}(100) 2 \times 1$ surface. Normal-emission photoelectron spectra were recorded in the photon-energy range 7-30 eV. Bulk direct transitions from the three uppermost valence bands to four different final bands are identified in the spectra. The experimental final bands are in good agreement with calculated conduction bands from a linearized augmented-plane-wave calculation. The experimental energies of the $X_{4}$, $\Gamma_{12}^{\prime}$, and $\Gamma_{2}^{\prime}$ critical points were found to be $-3.1 \pm 0.2,8.5 \pm 0.3$, and $15.8 \pm 0.5 \mathrm{eV}$, respectively. For further comparison between experiment and theory, normal-emission photoelectron spectra were calculated based on the three-step model. Most of the structures in the theoretical spectra are identified also in the experimental spectra. Apart from the direct transitions, also peaks due to nondirect and surface-umklapp scattered transitions were found in the experimental spectra, originating from $\mathbf{k}$ points with a high density of states, near the $X$ and $L$ symmetry points. At low photon energies a dispersive peak was also found, which could not be assigned to an allowed final band in the theoretical band structure for normal emission.
\end{abstract}

\section{INTRODUCTION}

The bulk band structures of semiconductors have been studied both experimentally and theoretically for many years. An important tool in recent experimental studies has been angle-resolved photoelectron spectroscopy (ARPES) in combination with tunable synchrotron-light sources. With ARPES, the dispersions of the bulk bands have been mapped for, e.g., $\mathrm{Si}^{1-5} \mathrm{Ge}^{2,6-10}$ and GaAs. ${ }^{11,12}$ which are the most extensively studied semiconductors. To map the initial bands, using the directtransition model, the final band(s) must first be determined, and vice versa. In most cases a free-electron final band has been used, since for sufficiently high photon energies, the final bands in photoemission can be approximated with free-electron bands. ${ }^{11}$

Considerably less experimental information has been obtained about the real, Bloch-type conduction bands. A reason for this may be the high density of conduction bands in the diamond- and zinc-blende-type crystals, but also the successful use of the free-electron approximation for high photon energies. For lower photon energies, $\sim 10-30 \mathrm{eV}$, the free-electron approximation is in general not applicable, and some conduction bands may be determined experimentally if the initial bands are known. Thus, for normal emission from the Ge(100) $2 \times 1$ (Ref. 8) and Ge(111) (Refs. 9 and 10) surfaces, the experimental final bands for the bulk structures were found to be in close agreement with calculated conduction bands. In contrast, bulk direct transitions in normal emission from the $\mathrm{Si}(111) 2 \times 1$ surface follow a free-electron final band also in the low photon-energy range. ${ }^{1}$ Important information about the lowest-lying conduction bands in $\mathrm{Ge}$ and $\mathrm{Si}$ has also been obtained with $\mathbf{k}$-resolved inverse photoemission on the $\mathrm{Ge}(111) 2 \times 1$ and $\mathrm{Si}(111) 2 \times 1$ surfaces. $^{13}$

In the present work, we have studied the bulk band structure of Si along the $\Delta$ symmetry line in the Brillouin zone (BZ) with angle-resolved photoemission from the $\mathrm{Si}(100) 2 \times 1$ surface. By the use of synchrotron radiation, normal-emission ARPES spectra in the photon-energy interval 7-30 eV were recorded. The data were analyzed with the help of a theoretical Si band structure, calculated with the linearized augmented-plane-wave (LAPW) method. Based on calculated initial bands, slightly modified in order to agree with experimental results, the final bands along the $\Delta$ line have been mapped. For further comparison between experiment and theory, theoretical bulk photoemission spectra were also calculated. Our results show that in this photon-energy range, the experimental final bands agree well with the calculated conduction bands, rather than with any free-electron band.

\section{THEORY}

In the analysis of the ARPES spectra, the bulk band structure from a LAPW calculation was used. ${ }^{14}$ The cal- 
culated band structure along $\Delta$ in the BZ, which corresponds to the [001] direction, is shown in Fig. 1, with the bands labeled according to their symmetries. The calculation was done within the density-functional theory, using the Hedin-Lundqvist ${ }^{15}$ local-density approximation (LDA) for correlation and exchange. The Si crystal potential was calculated self-consistently, using 89 plane waves and a convergence criterion of $0.1 \mathrm{mRy}$. To generate the band structure along line $\Delta, 181$ plane waves were used in order to accurately calculate the uppermost conduction bands in Fig. 1.

The band-structure calculation gave a fundamental band gap of $0.41 \mathrm{eV}$. This far too small value is a wellknown consequence of the LDA. The conduction bands

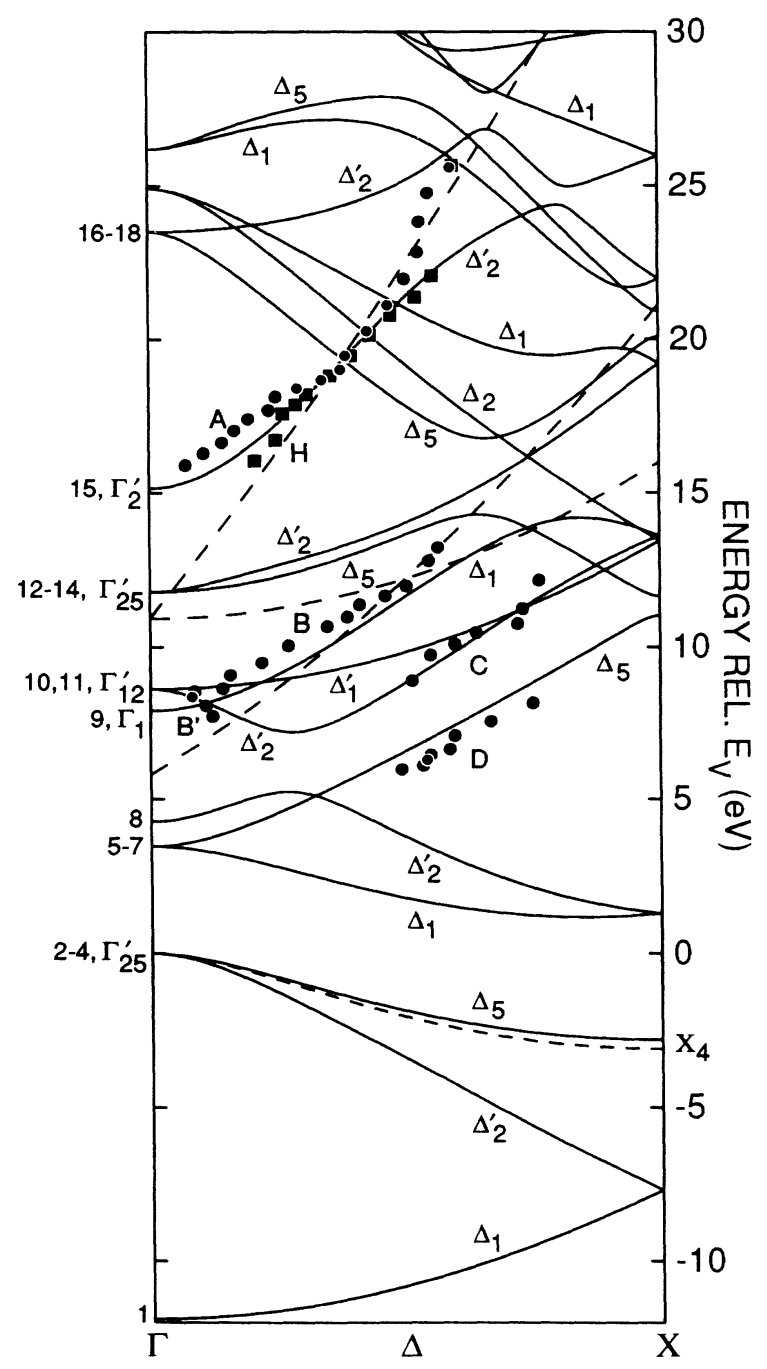

FIG. 1. Band structure of $\mathrm{Si}$ along the $\Delta$ symmetry line, calculated with the LAPW method. The numbers of the bands, according to their ordering near point $\Gamma$, are shown to the left. The symmetries of the bands are also indicated in the figure. Experimental direct transitions from VB 2 and VB 3,4 are shown with squares and circles, respectively. The labels for the experimental points are explained in the text. The dashed lines are the modified VB 3,4 and the free-electron bands for $\mathbf{k}+\mathbf{G}_{002}$, $\mathbf{k}+\mathbf{G}_{020}$, and $\mathbf{k}+\mathbf{G}_{111}$ using an inner potential of $-9.5 \mathrm{eV}$.

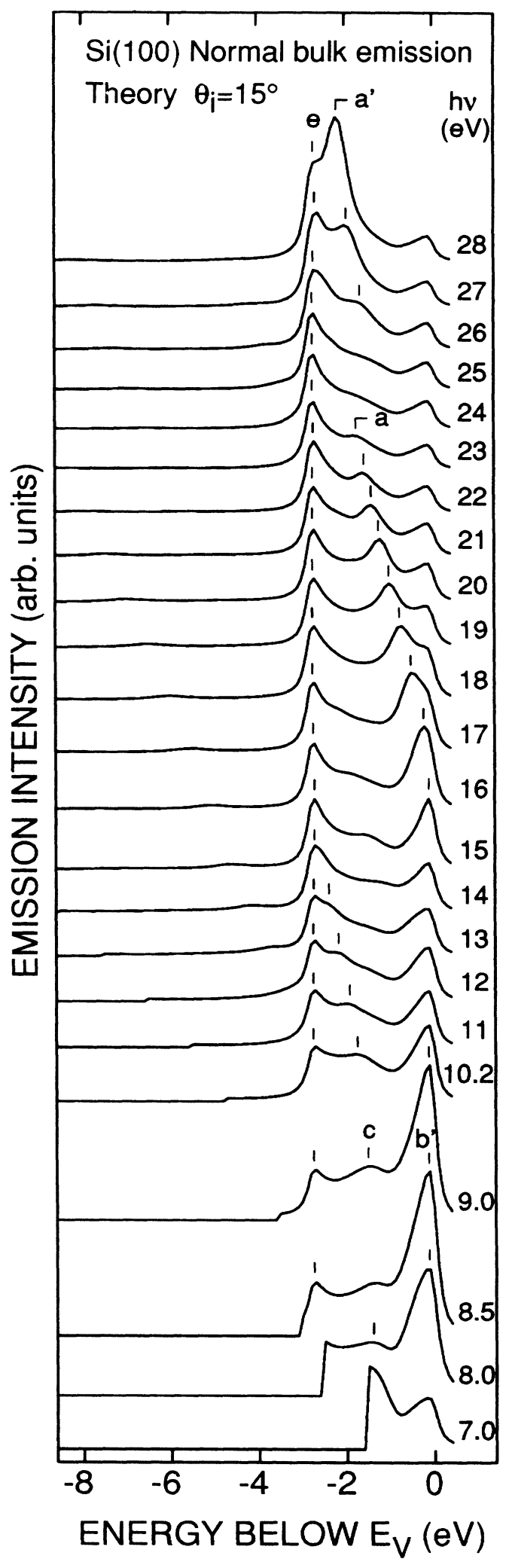

FIG. 2. Calculated bulk photoemission spectra in normal emission for various photon energies from $\mathrm{Si}(100)$. The orientation of the polarization vector corresponds to an incidence angle of $\theta_{t}=15^{\circ}$, referred to the surface normal. The labels are explained in the text. 
were therefore rigidly shifted to give the correct experimental band gap at $0 \mathrm{~K}: 1.17 \mathrm{eV}$. This procedure is known to give a good approximation to band structures calculated with many-body theory. ${ }^{14,16}$ This shift is thus

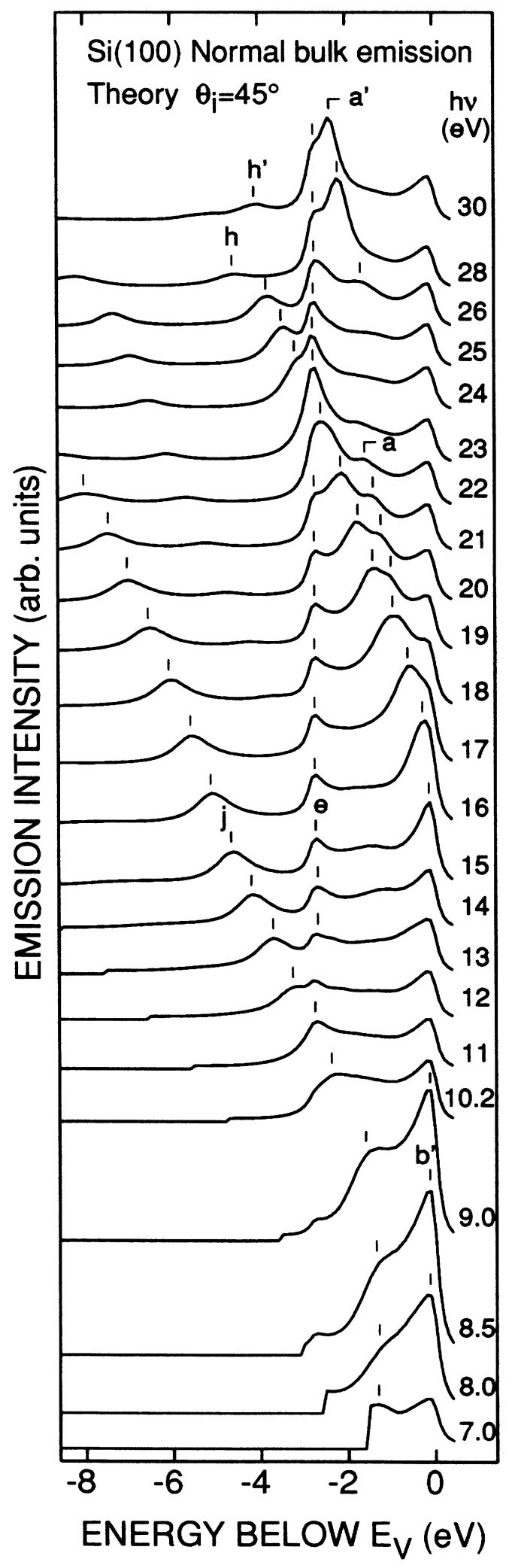

FIG. 3. Same as Fig. 2, but for $\theta_{1}=45^{\circ}$ :

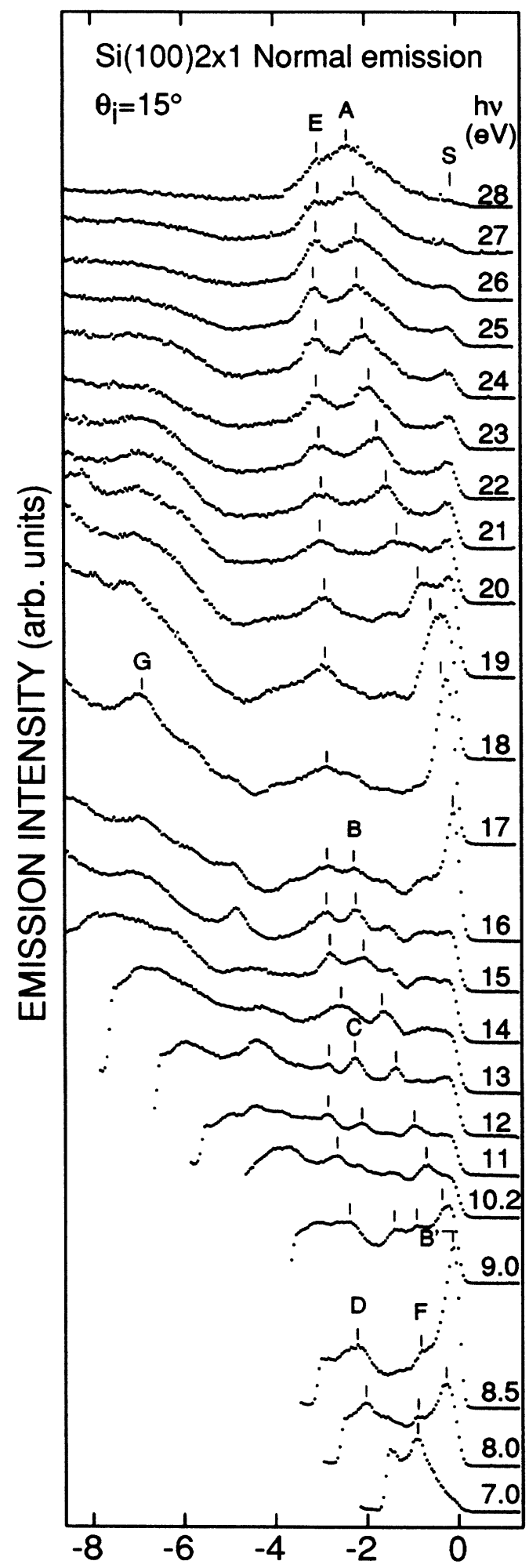

ENERGY BELOW E $\mathrm{V}_{\mathrm{V}}(\mathrm{eV})$

FIG. 4. Experimental normal-emission ARPES spectra recorded with various photon energies and $\theta_{1}=15^{\circ}$ from the $\mathrm{Si}(100) 2 \times 1$ surface. The labels are explained in the text. 
included in the energy values for the theoretical conduction bands in the discussion below, as well as in Fig. 1.

In order to more closely compare experiment to theory, bulk photoemission spectra were calculated using

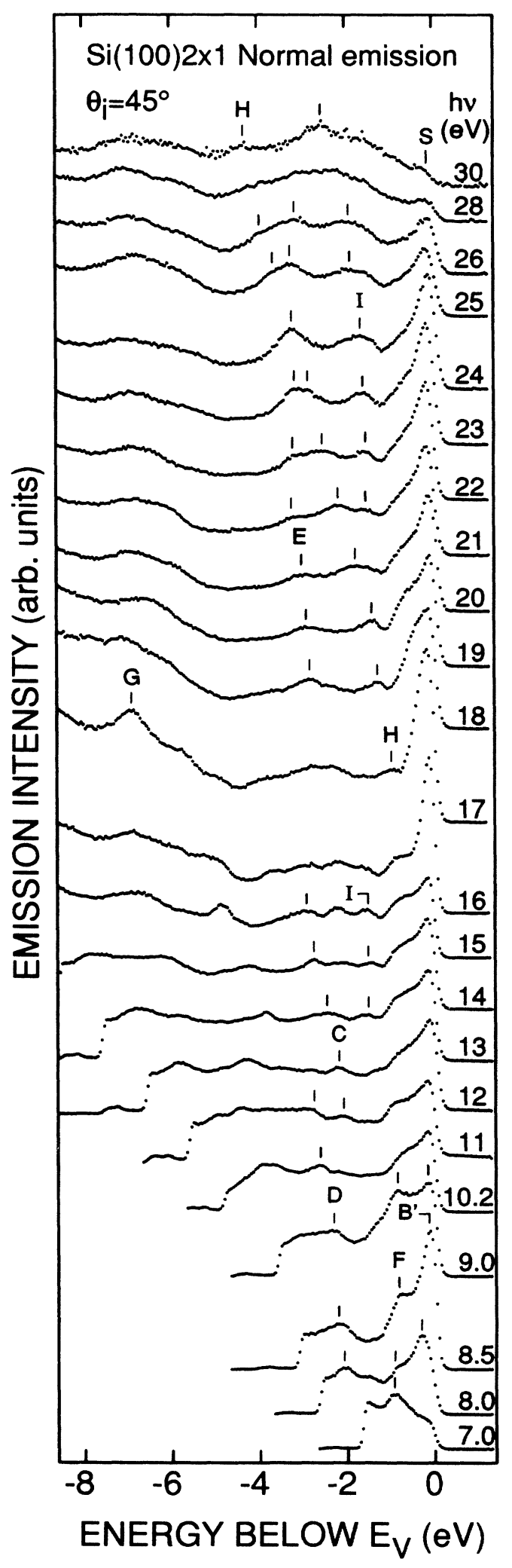

FIG. 5. Same as Fig. 4, but for $\theta_{1}=45^{\circ}$. a simplified three-step model. ${ }^{14}$ Thus only bulk direct transitions are contributing to the calculated spectra. The optical excitation was approximated with vertical transitions and the transition strength was given by the calculated dipole-matrix elements. The transport to the surface and the emission into vacuum was simulated by matching the bulk wave functions at the surface to the plane-wave solution in the vacuum. This matching was simplified by only including the terms in the plane-wave expansion of the wave functions for which $G_{\|}=0$. The potential change at the surface was then approximated by a simple step function. Initial- and final-state lifetime effects were included through Lorentzian broadening factors. The lifetime broadening was chosen so that the structures in the spectra are clearly visible and so that the spectra roughly resemble the experimental spectra in terms of the sharpness of the peaks. Theoretical spectra, calculated with initial- and final-state lifetime broadenings of 0.28 and $1.8 \mathrm{eV}$ full width at half maximum (FWHM), respectively, are shown in Figs. 2 and 3. The orientations of the polarization vector and photon energies are the same as for the corresponding experimental spectra in Figs. 4 and 5. The structures in the calculated spectra are discussed below.

\section{EXPERIMENTAL DETAILS}

The main ARPES experiments were performed in a VG ADES 400 spectrometer with polarized synchrotron radiation from the DORIS II storage ring at Hamburger Synchrotronstrahlungslabor (HASYLAB), Deutschen Elektronen-Synchrotron (DESY). ${ }^{17}$ The spectra presented here were recorded with the polarization vector parallel to the plane defined by the surface normal and the direction of the incident light ( $p$-polarized light). The energy resolution changed with photon energy, from $0.1 \mathrm{eV}$ for $h v=7 \mathrm{eV}$ to $0.3 \mathrm{eV}$ for $h v=28 \mathrm{eV}$. The angular resolution was $\pm 2^{\circ}$ and the base pressure of the system was $\sim 2 \times 10^{-10}$ Torr.

The sample was an $n$-type doped, mirror-polished $\mathrm{Si}(100)$ single crystal $(\rho=2 \Omega \mathrm{cm}$, arsenic, WackerChemitronic) with a size of $6 \times 17 \times 0.4 \mathrm{~mm}^{3}$. Before insertion into the vacuum chamber, it was precleaned using the etching procedure of Ishizaka and Shiraki. ${ }^{18}$ In ultrahigh vacuum it was cleaned by stepwise resistive heating up to $\sim 900^{\circ} \mathrm{C}$. A clean and well-ordered $2 \times 1$ surface was then obtained, which was evidenced by the sharp $2 \times 1$ low-energy electron diffraction (LEED) pattern and the strong emission from the contaminationsensitive surface states in the ARPES spectra. To maintain the clean surface, the sample was heated to $\sim 850-900^{\circ} \mathrm{C}$ approximately every $2.5 \mathrm{~h}$. During this time period, the decrease in emission intensity from the contamination-sensitive dangling-bond surface state was less than $25 \%$.

The Fermi-level $\left(E_{F}\right)$ position was determined by photoemission from the metallic ( $\mathrm{Ta}$ ) sample holder. To relate the measured electron energies to the valence-band maximum $\left(E_{V}\right)$, the value $E_{F}-E_{V}=0.4 \mathrm{eV}$ was used. This value for lightly $n$-type doped $\mathrm{Si}(100) 2 \times 1$ surfaces has been obtained by $\mathrm{Si} 2 p$ core-level photoemission studies of $\mathrm{Si}(100) 2 \times 1$ and $\mathrm{Si}(111) 7 \times 7$ surfaces. ${ }^{19}$ The value 
$E_{F}-E_{V}=0.63 \mathrm{eV}$ for $\mathrm{Si}(111) 7 \times 7$ was used as the reference. $^{20}$ Our experience is that at this low doping, the Fermi level on $\mathrm{Si}(100) 2 \times 1$ can shift a little, depending on the quality of the surface. We therefore estimate an error margin of $\pm 0.1 \mathrm{eV}$ for $E_{F}-E_{V}$.

Complementary ARPES experiments with hydrogen chemisorption on $\mathrm{Si}(100)$ were done in another chamber, using 21.2- and 16.85-eV photon energy from a resonance lamp. The experimental details from those experiments have been presented elsewhere. ${ }^{21}$

\section{RESULTS AND DISCUSSION}

ARPES normal-emission spectra, recorded with various photon energies on the $\mathrm{Si}(100) 2 \times 1$ surface, are shown in Figs. 4 and 5. For the spectra in Fig. 4, the angle of incidence $\left(\theta_{i}\right)$ is $15^{\circ}$, referred to the surface normal, and for the spectra in Fig. $5, \theta_{i}=45^{\circ}$. The intensities of the spectra are roughly normalized to an "average" background intensity in each spectrum, not counting the dispersing peaks that are interpreted as direct transitions. A general property of the spectra is the relatively small size of most of the bulk peaks, compared to the background, particularly for the $\theta_{i}=45^{\circ}$ spectra. In general, some background intensity is expected due to inelastic scattering and nondirect transitions. A recent detailed theoretical investigation of bulk transitions in a semiinfinite solid ${ }^{22}$ has shown that due to the existence of the surface, the bulk photoemission generally consists of two contributions of comparable magnitude. One contribution is the direct transitions, seen as strong peaks, and the second contribution is transitions where the $k_{\perp}$ component is not conserved, which manifests itself as a background intensity. The relatively small size of the bulk peaks, compared to the background, has been observed also in previous ARPES studies of the $\mathrm{Si}(100) 2 \times 1$ surface, ${ }^{2,5}$ and it has been suggested that the strong background emission may result from surface-reconstructioninduced strain ${ }^{2}$ or defects on the surface. ${ }^{5}$ Nevertheless, several structures that disperse with photon energy can be observed in the spectra in Figs. 4 and 5.

In the spectra in Fig. 4 , recorded with $\theta_{l}=15^{\circ}$, strong peaks emerge very close to the valence-band maximum $\left(E_{V}\right)$ at 8.5- and 16-eV photon energy. Going up in photon energy, these structures, denoted $A$ and $B$, disperse down to the initial energies $-2.4 \mathrm{eV}$ at $h v=28 \mathrm{eV}$ for $A$ and $-2.3 \mathrm{eV}$ at $h v=16 \mathrm{eV}$ for $B$ ( the peak at $h v=8.5$ $\mathrm{eV}$ is denoted $B^{\prime}$ for reasons discussed below). Two other dispersive structures, denoted $C$ and $D$ are observed at photon energies $8-12 \mathrm{eV}(D)$ and $11-15 \mathrm{eV}(C)$.

Prominent nondispersive features, seen over a large photon-energy range, are found at $\sim-3.1 \mathrm{eV}$, denoted $E$, and very close to $E_{V}$, denoted $S$. Other nondispersive structures are seen at $-0.8 \mathrm{eV}$ for $h v=7-9 \mathrm{eV}$ (denoted $F$ ), and at $\sim-6.9 \mathrm{eV}$ (denoted $G$ ) for several photon energies. The latter, very broad structure becomes particularly strong for $h v=17 \mathrm{eV}$. A few scattered peaks also occur in the spectra, mainly in the energy range $4-5 \mathrm{eV}$ below $E_{V}$.

In the spectra in Fig. 5, recorded with $\theta_{1}=45^{\circ}$, the most prominent structure is the nondispersive peak $S$ very close to $E_{V}$ that is seen over practically the entire photon-energy range. This structure is the well-known surface state associated with the dangling bonds on the $2 \times 1$ reconstructed surface (in normal emission, this state is, strictly speaking, a surface resonance). For several photon energies, this peak appears quite asymmetric, which has led to the suggestion that other surface resonances may exist at slightly lower initial energies. ${ }^{23,24}$ The $S$ peak and possibly the low-energy shoulder on this peak are the only structures identified as surface states in normal emission. Thus all other structures in the spectra are regarded as due to bulk emission.

Despite the dominating surface-state emission, sharp increases in the emission intensity at the $S$-peak position appear at $h v=8.5$ and $16 \mathrm{eV}$ also in these spectra. Close to the peak in the $17-\mathrm{eV}$ spectrum, a dispersive structure, denoted $H$, appears and disperses down to $\sim-4.3 \mathrm{eV}$ at $h v=30 \mathrm{eV}$. At lower photon energies, the dispersive structures $C$ and $D$ from the $\theta_{l}=15^{\circ}$ spectra are found also in the $\theta_{1}=45^{\circ}$ spectra. Similarly, the nondispersive structures $E, F$, and $G$ appear also in the $\theta_{i}=45^{\circ}$ spectra. At several photon energies, a peak, denoted $I$, is found at $\sim-1.5 \mathrm{eV}$. Above $h v=23 \mathrm{eV}$, this peak broadens and finally merges with the $E$ structure to a common, broad peak at $-2.5 \mathrm{eV}$ for $h v=30 \mathrm{eV}$.

The strong peaks that emerge as $E_{V}$ for $h v=8.5$ and $16 \mathrm{eV}$ are naturally interpreted as bulk direct transitions. However, the overlap in initial energy with the danglingbond surface state makes this assignment somewhat ambiguous. The nature of the peak in the photon-energy range 16-17 eV was studied in another ARPES experiment and discussed in Ref. 25, and it was found that the main contribution to this peak comes from the surface state, which shows a strong enhancement in emission intensity at $\sim 16-\mathrm{eV}$ photon energy. These results are illustrated in Fig. 6, where spectra recorded on the clean $\mathrm{Si}(100) 2 \times 1$ surface and the hydrogen-chemisorbed $2 \times 1: \mathrm{H}$ surface with $16.85-\mathrm{eV}$ photon energy are shown. From spectra at other emission angles or another photon energy, it is known that the hydrogen chemisorption completely removes the surface state and that bulk emission is in general not significantly affected by the adsorbate. $^{21}$ The remaining peak in the $2 \times 1: \mathrm{H}$ spectrum, which appears at a slightly lower initial energy than the surface state, thus corresponds to the bulk direct transition. However, the main intensity of the strong peak at the clean surface clearly comes from the surface state. The observed enhancement of the surface-state emission can be understood qualitatively in terms of the partly bulk origin of the dangling-bond surface state (which, as mentioned before, is actually a surface resonance near $\bar{\Gamma}$ ). As discussed theoretically in Refs. 26 and 27, photoemission from a surface state may become enhanced at photon energies where direct transitions exist from the bulk states closest in energy to the surface state. Similar resonances in the photoemission cross section of surface states have been observed previously on both metal surfaces $^{26-29}$ and semiconductor surfaces. ${ }^{30-31}$ For peak $B^{\prime}$ at $h v=8.5 \mathrm{eV}$, on the other hand, it was found in an earlier ARPES study that the main contribution to its intensity comes from a bulk direct transition. ${ }^{32}$ 
We start our analysis of the data by comparing the experimental and calculated spectra and thereafter the identified direct transitions are plotted in the calculated band structure. In doing that, it is very useful to know which transitions are allowed, according to the symmetry selection rules. For photoemission normal to the surface, i.e., along the [001] direction, the allowed final bands are $\Delta_{1}$ and $\Delta_{2}^{\prime}$ for diamond-type crystals, since these bands are invariant under the symmetry operations of the group $O_{h}^{7}$ (not including translations) that keep the [001] axis invariant. ${ }^{33,34}$ For the direct transitions, the dipole selection rules have been compiled by Eberhardt and Himpsel. ${ }^{35}$ In the present case, the possible initial bands are valence-band No. 2 (VB 2) and VB 3,4, with $\Delta_{2}^{\prime}$ and $\Delta_{5}$ symmetry, respectively. With the polarization vector

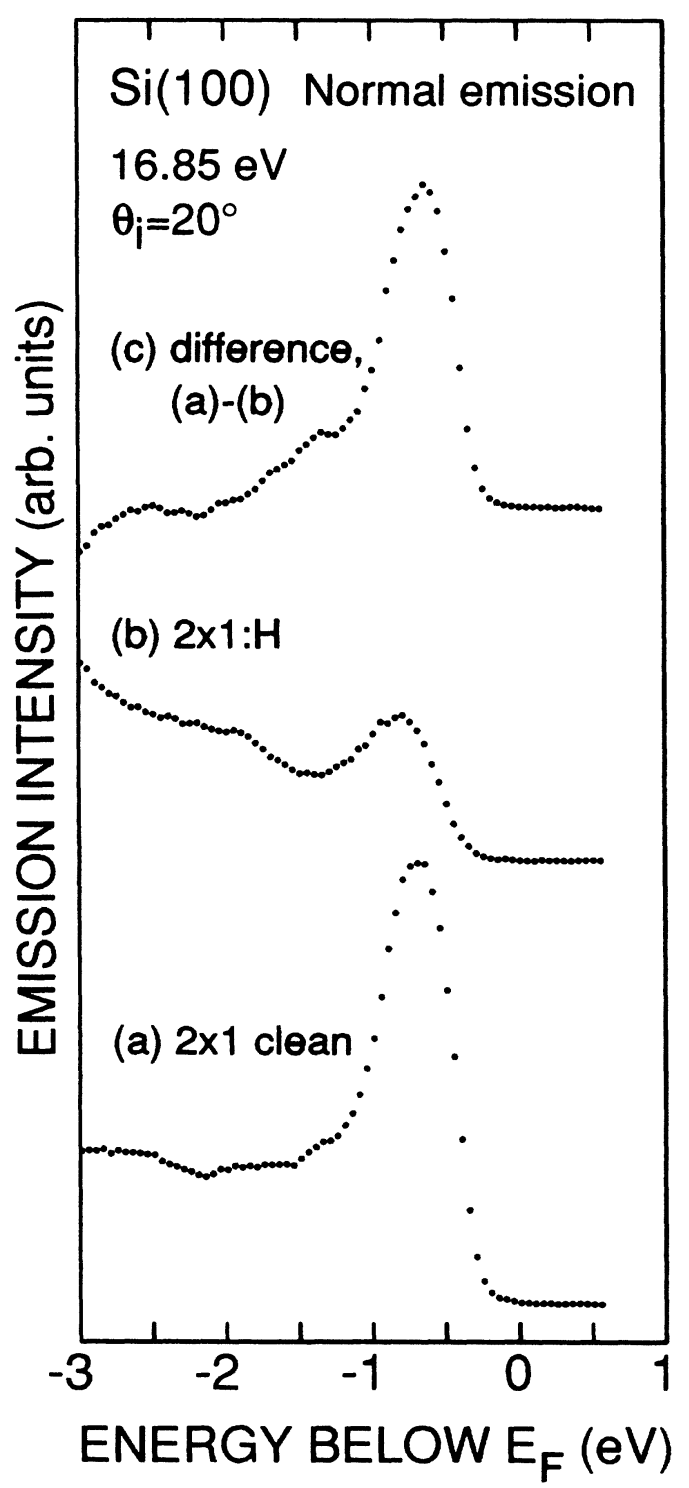

FIG. 6. Normal-emission spectra recorded with a photon energy of $16.85 \mathrm{eV}$ from a resonance lamp (Ref. 21) and $\theta_{1}=20^{\circ}$ (linearly polarized light) from (a) the $\mathrm{Si}(100) 2 \times 1$ (clean) surface, and (b) the $\mathrm{Si}(100) 2 \times 1: \mathrm{H}$ surface. (c) Difference spectrum between (a) and (b). parallel to the surface, the possible transitions to the allowed final bands are $\Delta_{5} \rightarrow \Delta_{2}^{\prime}$ and $\Delta_{5} \rightarrow \Delta_{1}$, whereas with the polarization vector perpendicular to the surface, the allowed transition is $\Delta_{2}^{\prime} \rightarrow \Delta_{2}^{\prime}$. Therefore, we expect to see emission mainly from VB 3,4 in the $\theta_{i}=15^{\circ}$ spectra, and contributions from both VB 2 and VB 3,4 in the $\theta_{1}=45^{\circ}$ spectra. In the calculated spectra, the selection rules are inherently satisfied in the matrix elements and the matching to free-electron wave functions at the surface.

Comparing the experimental spectra in Fig. 4, recorded with $\theta_{l}=15^{\circ}$, to the corresponding theoretical spectra in Fig. 2, several similarities can be observed. To simplify the comparison, structures in the theoretical spectra are labeled with the same letters (but lowercase) as corresponding features in the experimental spectra. The strong $B^{\prime}$ peak at $h v=8.5 \mathrm{eV}$ is well reproduced in the theoretical spectra $\left(b^{\prime}\right)$, whereas the peak at $E_{V}$ for $h v \sim 16 \mathrm{eV}$ is considerably smaller, in agreement with the established resonance behavior of the surface-state emission. The calculated structures $a, a^{\prime}, c$, and $e$ can be associated with the experimental structures $A, C$, and $E$. The peaks $a$ and $a^{\prime}$, which are transitions to two different $\Delta_{2}^{\prime}$ final bands, thus correspond to the apparent single structure $A$ in the experimental spectra. In the $\theta_{1}=45^{\circ}$ spectra in Figs. 3 and 5, similar identifications can be done. The $B^{\prime}$ and $b^{\prime}$ peaks at $h v=8.5 \mathrm{eV}$ appear slightly broader than for $\theta_{i}=15^{\circ}$. Structures $e, h$, and $h^{\prime}$ can be identified as the experimental $E$ and $H$ structures. Similar to the case of $a^{\prime}$ and $A$, the $H$ peak in the $h v=30-\mathrm{eV}$ spectrum corresponds to the $h^{\prime}$ peak, which is a transition to the highest-lying $\Delta_{2}^{\prime}$ band in Fig. 1. At the highest photon energies, the $a^{\prime}$ transition dominates in both the $\theta_{i}=15^{\circ}$ and $45^{\circ}$ theoretical spectra, which also appears to be consistent with the experimental spectra.

Some significant discrepancies between the experimental and calculated spectra can also be observed. In the $\theta_{1}=15^{\circ}$ spectra, the experimental dispersive structures $B$ and $D$ are not reproduced in the calculated spectra. In the $\theta_{l}=45^{\circ}$ spectra, the strong calculated structure $j$ has not been clearly identified in the experimental spectra, although there exist peaks at $\sim 4-5 \mathrm{eV}$ below $E_{V}$ in the 13-, 14-, and 15-eV spectra in Fig. 5, which may correspond to $j$. For peaks $B$ and $j$, these discrepancies may be caused by the simplified matching of the Bloch-type wave functions to free-electron waves at the surface plane, which may give incorrect emission intensities. In the $\theta_{1}=45^{\circ}$ spectra, the faint appearance of most bulk peaks is an additional difficulty. The nature of structure $D$ is uncertain, as discussed below. Another difference between experiment and theory is the appearance of the nondispersive, broad structure $G$ at $\sim-6.9 \mathrm{eV}$. Finally, a small peak is observed in the theoretical spectra near $E_{V}$ throughout the photon-energy range, looking conspicuously similar to the surface state. However, only bulk emission is included in the calculated spectra and the appearance of a nondispersive bulk peak near $E_{V}$ is not consistent with earlier experimental results on the $\mathrm{Si}(100) 2 \times 1: \mathrm{H}$ surface, see e.g., Ref. 21 . This peak appears to be an artifact of the calculation caused by the use of a constant Lorentzian lifetime broadening for the 
valence bands. This approximation becomes unrealistic near $E_{V}$, where the broadening should approach zero.

Of particular interest are the nondispersive structures $E$ and $e$ at -3.1 and $-2.8 \mathrm{eV}$, respectively. Bulk peaks that do not disperse with photon energy are often regarded as nondirect transitions from regions in the $\mathrm{BZ}$ with a high density of states (DOS) in the valence bands. In the present case, the theoretical $e$ peak originates from the almost flat part of VB 3,4 near the $X$ symmetry point and the energy of peak $e$ coincides with the calculated $X_{4}$ value $(-2.81 \mathrm{eV})$. This DOS effect in the calculation is due to the lifetime broadening, which may be regarded as a small relaxation of the $k_{\perp}$ conservation. By assigning the experimental structure $E$ to $e$, we determine the experimental value of the $X_{4}$ energy to be $-3.1 \mathrm{eV}$, in good agreement with earlier experimental results. $2,3,5$

The final bands of the identified experimental direct transitions are plotted in the band structure in Fig. 1. The final-band energies are obtained by adding the photon energy to the initial energy of each peak. The $k_{\perp}$ value of each transition is obtained from the calculated valence bands by observing where along $\Delta$ the initial energy of the peak coincides with the identified initial band. In doing that, a modified dispersion has been used for VB 3,4 , as illustrated in Fig. 1 by the dashed line. The band energies have been multiplied by a factor that gives the experimental value $-3.1 \mathrm{eV}$ for $X_{4}$.

The identification of the initial bands is simplified by the dipole selection rules and the calculated spectra. Thus the structures $A, B$, and $C$ are identified as direct transitions from VB 3,4 $\left(\Delta_{5}\right)$. The plotted final states in Fig. 1 follow fairly well the dispersions of the conduction bands (CB) No. $15\left(\Delta_{2}^{\prime}\right)$, CB $9\left(\Delta_{1}\right)$, and CB $10\left(\Delta_{2}^{\prime}\right)$, respectively. The structure $H$ from the $45^{\circ}$ spectra is identified as a direct transition from VB $2\left(\Delta_{2}^{\prime}\right)$ to CB 15 $\left(\Delta_{2}^{\prime}\right)$, as seen in Fig. 1. At the highest photon energies, both structures $A$ and $H$ deviate from CB 15 and reach the uppermost $\Delta_{2}^{\prime}$ band, as predicted by the theoretical spectra. Also near $\Gamma$, the $A$ and $H$ structures appear to deviate slightly from CB 15 . For the $A$ structure, this may be caused by the interference from the strong surface-state emission, which makes it difficult to determine the initial energy of the $A$ peak near $\Gamma$.

For comparison, free-electron final bands are plotted in Fig. 1 using an inner potential of $-9.5 \mathrm{eV}$. It is clear that the final bands in general are better described by the calculated band structure, although some transitions ( $A$, $B$, and $H$ ), at least partly, can be explained by freeelectron bands.

An interesting observation is that direct transitions are found to all $\Delta_{2}^{\prime}$ conduction bands above the vacuum level (in particular CB 15), except CB 14. In the theoretical spectra, transitions to $\mathrm{CB} 14$ can be found, but they are faint. A similar effect was found in the ARPES study of Nelson et al., ${ }^{8}$ of $\mathrm{Ge}(100) 2 \times 1$. The conduction band in Ge along $\Delta$ corresponding to CB 15 (the band structures of $\mathrm{Si}$ and Ge are very similar) carried nearly all the photonemission current from the observed bulk direct transitions in this photon-energy range. This was explained by the high [002] plane-wave character of this band. In the case of $\mathrm{Si}(100)$, the absence of strong transitions to CB 14 appears to be a combination of smaller matrix elements, compared to $\mathrm{CB} 15$, and the effect of the matching at the surface.

A detailed inspection of the strong peaks at $E_{V}$ for photon energies around 8.5 and $16 \mathrm{eV}$ allows us to determine the values of the $\Gamma_{12}^{\prime}$ and $\Gamma_{2}^{\prime}$ critical points to $8.5 \pm 0.3 \mathrm{eV}$ and $15.8 \pm 0.5 \mathrm{eV}$, respectively. The assignment of the $8.5-\mathrm{eV}$ peak $\left(B^{\prime}\right)$ to a transition to $\Gamma_{12}^{\prime}$ is based on the dipole selection rules, ${ }^{35}$ where the transition $\Gamma_{25}^{\prime} \rightarrow \Gamma_{1}$ is forbidden. Therefore, the $B^{\prime}$ peak does not belong to the $B$ structure, which is a transition to the $\Delta_{1}$ band. The experimental values for the $\Gamma_{12}^{\prime}$ and $\Gamma_{2}^{\prime}$ critical points are in good agreement with the calculated values: 8.64 and $15.15 \mathrm{eV}$, respectively. In Fig. 1, the plotted points do not reach $\Gamma$ at 8.5 and $15.8 \mathrm{eV}$. However, the distance in $\mathbf{k}$ space from $\Gamma$ is within the uncertainty in the experimental $k_{1}$ values, which is relatively large near $\Gamma$ due to the flatness of the valence-band dispersions at this point.

The dispersive structure $D$ is seen in the experimental spectra for both $\theta_{1}=15^{\circ}$ and $45^{\circ}$. Assuming it is a direct transition along $\Delta$ from VB 3,4 , a final band is found close to CB 6,7 $\left(\Delta_{5}\right)$, as illustrated in Fig. 1. However, these bands are not allowed final bands for normal emission, according to the selection rules. Also the nearly identical appearance of this structure in both spectra series is inconsistent with the selection rules. On the other hand, the clear dispersion with photon energy is not consistent with a valence-band DOS assignment. Also surface-umklapp scattered direct transitions (discussed below) are ruled out as an explanation for $D$. We conclude that we have no explanation that is consistent with the symmetry selection rules and the direct-transition model for this structure.

The peak denoted $F$, seen at the lowest photon energies, is not well reproduced in the calculated spectra. Yet we find that the most reasonable interpretation of this peak is that it is a transition from CB 3,4 to the minimum of CB $10\left(\Delta_{2}^{\prime}\right)$. In the $\theta_{t}=15^{\circ}$ spectra, it should then connect with structure $C$, which also appears to happen. In the $\theta_{1}=45^{\circ}$ spectra, its intensity is enhanced, which is consistent with the broad $b^{\prime}$ peak in the theoretical spectra. This broadening is caused by transitions from VB 2 $\left(\Delta_{2}^{\prime}\right)$ to CB $10\left(\Delta_{2}^{\prime}\right)$. These bands are almost parallel near $\Gamma$ with an energy distance of $\sim 8.5 \mathrm{eV}$, which explains the broad $b^{\prime}$ peak at $h v=8.5 \mathrm{eV}$.

Because of the surface reconstruction, one also has to consider possible bulk contributions that are umklapp scattered into normal emission at the surface, involving a reciprocal-lattice vector of the surface mesh. For the Si $(100) 2 \times 1$ surface, bulk transitions along a line in the BZ parallel to the surface normal and passing through the $L$ symmetry point can be surface-umklapp scattered into normal emission. Our calculations show that the dispersion of the uppermost valence band is almost flat along this line, which gives a high DOS at the energy of the $L_{3}^{\prime}$ critical point. In the experimental $\theta_{i}=45^{\circ}$ spectra, peaks are found at $\sim-1.5 \mathrm{eV}$ for several photon energies, e.g., 13-15 eV and 21-24 eV (denoted I), which may be attributed to surface-umklapp scattered transitions from the above-mentioned line through $L$. The 
value $-1.5 \mathrm{eV}$ for the $L_{3}^{\prime}$ point agrees well with earlier experimental results, ${ }^{1,30}$ whereas the calculated value is too small: $-1.14 \mathrm{eV}$. The broad, nondispersive structure $G$ at $\sim-6.9 \mathrm{eV}$ may also be due to surface-umklapp scattering, or, alternatively, to a three-dimensional DOS contribution. In a recent calculation, ${ }^{36}$ a sharp peak was found in the total DOS of Si slightly above $(\sim 0.2 \mathrm{eV})$ the $L_{1}$ critical point. The calculated value for $L_{1}$ is -6.97 $\mathrm{eV}$ in the present work, in good agreement with the experimental value in either case.

The bulk band structure of Si along the $\Delta$ symmetry line has previously been studied from ARPES on Si(100) surfaces by Wachs et al. ${ }^{2}$ and Rich et al. ${ }^{3}$ In the latter study, an Sb-terminated surface was used in order to reduce strain induced by the surface reconstruction. Higher photon energies than in the present work were used and the valence bands were mapped, using freeelectron final bands. However, at low photon energies, dispersive structures were found that could not be analyzed with free-electron final bands. Comparing those data to the present data, we observe that the strong peak denoted $E$ in Ref. 2 and the peak denoted $D 2$ in Ref. 3 appear to coincide with structures $H$ and $h$ in the present work. Peak $D$ in Ref. 2 appears to be the same as our peak $I$, whereas structure $F$ in Ref. 2 has no corresponding structure in either our experimental or our calculated spectra. Normal-emission ARPES bulk studies on $\mathrm{Si}(100) 2 \times 1$ have also been reported by Koke et al., ${ }^{4}$ however, with a very limited data set.

\section{SUMMARY}

The bulk band structure of silicon has been studied with angle-resolved photoemission from the $\mathrm{Si}(100) 2 \times 1$ surface. Normal-emission spectra have been recorded using synchrotron radiation in the photon-energy range $7-30 \mathrm{eV}$. The data have been analyzed with the help of a theoretical Si band structure, calculated with the LAPW method. For further comparison between experiment and theory, theoretical bulk photoemission spectra were calculated using a simplified three-step model. A good agreement between the experimental and theoretical spectra was found. As expected, free-electron final bands are not applicable in this photon-energy range. Instead, direct transitions to four different final bands along the $\Delta$ line in the Brillouin zone were identified in the experimental spectra. The experimental final bands are in good agreement with calculated conduction bands of the correct symmetry, as determined by the symmetry selection rules. At low photon energies, a dispersive peak was also found, which could not be assigned to any allowed final band for normal emission in the theoretical band structure. The energies of several critical points in the bulk band structure have been determined.

\section{ACKNOWLEDGMENTS}

The authors wish to thank Professor Göran Hansson for stimulating and helpful discussions, and the staff at HASYLAB for their help and support. This work was supported by the Swedish Natural Science Research Council.
*Present address: IBM Research Division, Zurich Research Laboratory, CH-8803 Rüschlikon, Switzerland.

${ }^{\dagger}$ Present address: National Supercomputer Center, Linköping University, S-58183 Linköping, Sweden.

${ }^{1}$ R. I. G. Uhrberg, G. V. Hansson, U. O. Karlsson, J. M. Nicholls, P. E. S. Persson, S. A. Flodström, R. Engelhardt, and E.-E. Koch, Phys. Rev. Lett. 52, 2265 (1984); Phys. Rev. B 31, 3795 (1985).

${ }^{2}$ A. L. Wachs, T. Miller, T. C. Hsieh, A. P. Shapiro, and T.-C. Chiang, Phys. Rev. B 32, 2326 (1985).

${ }^{3}$ D. H. Rich, T. Miller, G. E. Franklin, and T.-C. Chiang, Phys. Rev. B 39, 1438 (1989); D. H. Rich, G. E. Franklin, F. M. Leibsle, T. Miller, and T.-C. Chiang, ibid. 40, 11804 (1989).

${ }^{4}$ P. Koke, A. Goldmann, W. Mönch, G. Wolfgarten, and J. Pollmann, Surf. Sci. 152/153, 1001 (1985).

${ }^{5}$ A. Goldmann, P. Koke, W. Mönch, G. Wolfgarten, and J. Pollmann, Surf. Sci. 169, 438 (1986).

${ }^{6}$ T. C. Hsieh, T. Miller, and T.-C. Chiang, Phys. Rev. B 30, 7005 (1984).

${ }^{7}$ R. D. Bringans and H. Höchst, Phys. Rev. B 25, 1081 (1982).

${ }^{8}$ J. G. Nelson, W. J. Gignac, R. S. Williams, S. W. Robey, J. G. Tobin, and D. A. Shirley, Surf. Sci. 131, 290 (1983).

${ }^{9}$ J. M. Nicholls, G. V. Hansson, U. O. Karlsson, P. E. S. Persson, R. I. G. Uhrberg, R. Engelhardt, S. A. Flodström, and E.-E. Koch, Phys. Rev. B 32, 6663 (1985).

${ }^{10}$ R. D. Bringans, R. I. G. Uhrberg, and R. Z. Bachrach, Phys. Rev. B 34, 2373 (1986).

${ }^{11}$ T.-C. Chiang, J. A. Knapp, M. Aono, and D. E. Eastman,
Phys. Rev. B 21, 3513 (1980).

${ }^{12}$ T.-C. Chiang, R. Ludeke, M. Aono, G. Landgren, F. J. Himpsel, and D. E. Eastman, Phys. Rev. B 27, 4770 (1983).

${ }^{13}$ D. Straub, L. Ley, and F. J. Himpsel, Phys. Rev. Lett. 54, 142 (1985); Phys. Rev. B 33, 2607 (1986).

${ }^{14}$ P. E. S. Persson, Ph.D. thesis, Linköping University, 1986, dissertation No. 147.

${ }^{15}$ L. Hedin and B. Lundqvist, J. Phys. C 4, 2064 (1971).

${ }^{16}$ M. S. Hybertsen and S. G. Louie, Phys. Rev. Lett. 55, 1418 (1985).

${ }^{17}$ C. A. Feldmann, R. Engelhardt, T. Permien, E.-E. Koch, and V. Saile, Nucl. Instrum. Methods 208, 785 (1983).

${ }^{18}$ A. Ishizaka and Y. Shiraki, J. Electrochem. Soc. 133, 666 (1986).

${ }^{19}$ R. I. G. Uhrberg (unpublished).

${ }^{20}$ F. J. Himpsel, G. Hollinger, and R. A. Pollak, Phys. Rev. B 28, 7014 (1983).

${ }^{21}$ L. S. O. Johansson, R. I. G. Uhrberg, and G. V. Hansson, Surf. Sci. 189/190, 479 (1987).

${ }^{22}$ B. C. Meyers and T. E. Feuchtwang, Phys. Rev. B 35, 3697 (1987).

${ }^{23}$ F. J. Himpsel and D. E. Eastman, J. Vac. Sci. Technol. 16, 1297 (1979).

${ }^{24}$ L. S. O. Johansson, R. I. G. Uhrberg, P. Mårtensson, and G. V. Hansson, Phys. Rev. B 42, 1305 (1990).

${ }^{25}$ L. S. O. Johansson, E. Landemark, G. V. Hansson, and R. I. G. Uhrberg, Surf. Sci. 211/212, 578 (1989).

${ }^{26}$ S. G. Louie, P. Thiry, R. Pinchaux, Y. Petroff, D. Chandesris, 
and J. Lecante, Phys. Rev. Lett. 44, 549 (1980).

${ }^{27}$ T. C. Hsieh, P. John, T. Miller, and T.-C. Chiang, Phys. Rev. B 35, 3728 (1987).

${ }^{28}$ H. J. Levinson, F. Greuter, and E. W. Plummer, Phys. Rev. B 27, 727 (1983).

${ }^{29}$ S. D. Kevan, N. G. Stoffel, and N. V. Smith, Phys. Rev. B 32, 4956 (1985).

${ }^{30}$ F. J. Himpsel, P. Heimann, and D. E. Eastman, Phys. Rev. B 24, 2003 (1981).

${ }^{31}$ L. Sorba, V. Hinkel, H. U. Middelmann, and K. Horn, Phys.
Rev. B 36, 8075 (1987).

${ }^{32}$ R. I. G. Uhrberg, G. V. Hansson, J. M. Nicholls, and S. A. Flodström, Phys. Rev. B 24, 4684 (1981); and R. I. G. Uhrberg (unpublished).

${ }^{33}$ J. Hermanson, Solid State Commun. 22, 9 (1977).

${ }^{34}$ J. C. Slater, Symmetry and Energy Bands in Crystals (Dover, New York, 1972), p. 372.

${ }^{35}$ W. Eberhardt and F. J. Himpsel, Phys. Rev. B 21, 5572 (1980).

${ }^{36}$ J. R. Chelikowsky, T. J. Wagener, J. H. Weaver, and A. Jin, Phys. Rev. B 40, 9644 (1989). 